\title{
Postoperative troponin increases after noncardiac surgery are associated with raised neurofilament light: a prospective observational cohort study.
}

\author{
Robert D. Sanders ${ }^{1,2^{*}}$ \\ Lenka Craigova $^{3}$ \\ Benjamin Schessler ${ }^{4}$ \\ Cameron Casey ${ }^{4}$ \\ Marissa White ${ }^{4}$ \\ Margaret Parker ${ }^{4}$ \\ David Kunkel ${ }^{4}$ \\ Kaj Blennow ${ }^{5}$ \\ Henrik Zetterberg ${ }^{5-7}$ \\ Robert A. Pearce ${ }^{4}$ \\ Richard Lennertz ${ }^{4}$
}

Short title: Myocardial injury \& Neurotoxicity

\section{Affiliations}

1. University of Sydney, Sydney, Australia

2. Department of Anaesthetics, Royal Prince Alfred Hospital, Building 89 Level 4 / Lambie Dew Drive, Camperdown, NSW, 2050, Australia

3. Medical Student Training Program, University of Wisconsin, Madison, USA

4. Department of Anesthesiology, University of Wisconsin, Madison, USA

5. Department of Psychiatry and Neurochemistry, Institute of Neuroscience and Physiology, the Sahlgrenska Academy at the University of Gothenburg, Mölndal, Sweden 
6. Clinical Neurochemistry Laboratory, Sahlgrenska University Hospital, Mölndal, Sweden

7. Department of Neurodegenerative Disease, UCL Institute of Neurology, Queen Square, London, United Kingdom

8. UK Dementia Research Institute at UCL, London, United Kingdom

${ }^{*}$ Corresponding author: Prof Robert D. Sanders, Department of Anaesthetics, Royal Prince Alfred Hospital, Building 89 Level 4 / Lambie Dew Drive, Camperdown, NSW, 2050, Australia. robert.sanders@sydney.edu.au

Word count

Title:

Abstract:

Article: 2 


\section{Abstract}

Background: Myocardial and neuronal injury occur commonly after noncardiac surgery. We examined whether patients who had perioperative myocardial injury (PMI) also incurred neuronal injury, and whether myocardial and neuronal injury were associated with similar changes in inflammatory markers or overlapping clinical predictors.

Methods: A total of 114 individuals $>65 \mathrm{yr}$ old were recruited from two ongoing perioperative cohort studies (NCT02926417; NCT03124303). Plasma samples were collected before and daily after surgery to process assays for troponin I (PMI), neurofilament light ( $\mathrm{NfL}$; neuronal injury) and multiplexed plasma cytokines (inflammation). The primary outcome was the change in $\mathrm{NfL}$ in individuals with PMI (>40 pg ml-1 increase in troponin above preoperative values). We conducted logistic regression to identify if there were shared clinical predictors for myocardial and neuronal injury.

Results: Ninety-six patients had paired NfL and troponin data. Twenty-three of 94 subjects (24\%) with PMI had greater increases in NfL (median [inter-quartile range, IQR]: 29 pg ml-1 [3-95 pg ml1]; 2.8-fold increase) compared with subjects with no troponin increase (8 pg ml-1 [3-20]; 1.3fold increase; $P=0.008)$. $P M I$ was associated with increased interleukin (IL)-1ra ( $P=0.005)$, IL-2 $(P=0.045), I L-8(P=0.002)$, and IL-10 $(P<0.001)$. Logistic regression showed that intraoperative hypotension was associated with $\mathrm{PMI}(\mathrm{P}=0.043)$. Preoperative stroke $(P=0.041)$ and blood loss $(P=0.002)$, but not intraoperative hypotension, were associated with increased NfL. 
Conclusions: Postoperative troponin increases were associated with changes in NfL and inflammatory cytokines. Increases in troponin, but not $\mathrm{NfL}$, were associated with intraoperative hypotension, suggesting differences in the mechanisms contributing to neuronal and myocardial injury.

Keywords: delirium; inflammation; injury; myocardial; neuronal; surgery; troponin. 


\section{Introduction}

Several studies have shown a correlation between postoperative troponin levels and mortality, after cardiac and noncardiac surgeries ${ }^{1-3}$. While overt injury associated with clinical symptoms remains uncommon, plasma biomarkers may identify lesser injuries that do not develop clinical symptoms. Indeed, postoperative increases in cardiac troponin in the absence of electrocardiogram changes are more common than those associated with electrocardiogram changes, and yet have been associated with similar risks of postoperative mortality ${ }^{1,3,4}$. Whether this is causally related to myocardial ischaemia or representative of a more generalized predisposition to perioperative complications needs further enquiry ${ }^{5,6}$. Similarly, neurofilament light $(\mathrm{NfL})$ has been identified as a potential plasma biomarker of neuronal injury. A postoperative rise in $\mathrm{NfL}$ has been associated with postoperative delirium ${ }^{7}$, as has covert stroke diagnosed on magnetic resonance imaging ${ }^{8}$, which suggests that neuronal injury may be an important component of the pathogenesis of delirium.

Given the importance of perioperative myocardial injury after non-cardiac surgery (MINS) ${ }^{3,5,9}$ and neuronal injury, we were interested in exploring whether there was common underlying aetiology. Therefore, we tested whether patients with troponin positive events would also exhibit higher levels of the neuronal injury biomarker, NfL. Second, we tested whether cardiac and neuronal injury were associated with similar changes in inflammatory markers or overlapping clinical predictors. 
Inflammation has been associated with cardiac injury in the setting of sepsis or cardiac surgery $^{10,11}$. Despite associations between myocardial injury and inflammation, we are unaware of studies probing associations after non-cardiac surgery. Recently, it was shown that preoperative inflammation may be associated with postoperative myocardial injury ${ }^{9}$. Similarly, our previous research suggests that perioperative inflammation plays an important role in neuronal injury ${ }^{7}$. We hypothesized that if there is a common inflammatory aetiology of neuronal and cardiac injury, then similar inflammatory cytokine profiles would be associated with neurofilament light and troponin rises. On the other hand, myocardial injury has been associated with intraoperative hypotension ${ }^{12,13}$ potentially contrasting with our recent findings with $\mathrm{NfL}^{7}$. Therefore, we sought to analyze, in our dataset, whether intraoperative hypotension was associated with troponin rises and hence identify differing mechanisms of myocardial and neuronal injury.

Our primary outcome, followed several leading studies in the field in diagnosing troponin positive patients (TPP) based on solely postoperative troponin values ${ }^{3}$. However, our work with NfL, has concentrated on the change from preoperative values (to detect the changes attributable to perioperative care) and therefore, we also analysed whether significant changes in troponin were associated with NfL rises. 


\section{Methods}

The data are derived from two ongoing prospective perioperative cohort studies registered with ClinicalTrials.gov (NCT02926417 and NCT03124303) and approved by the University of Wisconsin-Madison Institutional Review Board (2015-0960 and 2015-0374). 114 adult patients were recruited who were scheduled for major elective non-intracranial, non-cardiac surgery which was defined as requiring at least a two-day hospital stay (described in our recent publication on $\mathrm{NfL}^{7}$ ). All patients received general anesthesia during surgery. STROBE diagram is available in Figure 1 and the inclusion and exclusion criteria are presented in Supplementary Table 1. Blood draws were taken preoperatively and for first (up to) four postoperative hospital days if possible. Plasma samples were collected in EDTA-containing tubes preoperatively, and in the morning (06.00-10.00) of each postoperative day, spun and stored at $-80^{\circ} \mathrm{C}$.

\section{Troponin Assay}

Samples were then sent as a batch for troponin Tnl measured by MILLIPLEX ${ }^{\circledR}$ MAP Human Cardiovascular Disease Magnetic Bead single plex (Millipore,) completed by Eve technology, Canada. This assay shows $<10 \%$ intra-assay, and $<20 \%$ inter-assay coefficients of variation (precision) and $100 \%$ recovery of spiked standards (accuracy).

All 114 patients had at least one postoperative blood sample tested for troponin. We followed the literature in binarizing the troponin data into positive and negative status as many data points showed that troponin was not detected ${ }^{3}$. To fit the data as continuous variables for descriptive 
(non-statistical purposes), undetected values were ascribed the lowest limit of detection on the assay $(0.1 \mathrm{ng} / \mathrm{ml})$. As, the data was heavily skewed with many values under the lower limit of detection, we did not analyse troponin as continuous data variable. Samples were designated as positive if they exceeded a threshold of $40 \mathrm{ng} / \mathrm{ml}$ based on our local hospital assay. This level was chosen to allow corroboration of postoperative troponin values derived clinically with the research assay. Our primary outcome involved a binarized outcome of troponin positive patients (TPP) based on just postoperative samples. We extended this by looking at the change in troponin values based on an increase of interest in troponin of $40 \mathrm{ng} / \mathrm{ml}$.

\section{Cytokine and Neurofilament Light Assays}

A cytokine multiplex assay was completed for interleukin 1 (IL-1) beta, IL-1 receptor antagonist, IL-2, IL-4, IL-6, IL-8, IL-10, IL-12, monocyte chemoattractant protein 1 (MCP-1) and tumor necrosis factor-alpha (TNFa) (Eve technology, Canada). NfL concentration was also measured using a Simoa method, as previously described in detail ${ }^{14}$. All biomarker data were log-transformed to correct the strong rightward skew. In total, 103 participants had both preoperative and POD1 blood samples analyzable for NfL. When the difference between preoperative and POD1 samples was calculated, we took the difference of the log-transformed data.

\section{Delirium Assessments}


Preoperatively, and twice daily postoperatively, participants underwent delirium assessments with the Confusion Assessment Method (CAM)/3D-CAM, or the CAM-ICU if the patient was intubated. Delirium severity was assessed with the validated Delirium Rating Scale-98 (DRS).

\section{Statistical Analysis}

The sample size was determined based on prior data suggesting a $20 \%$ incidence in MINS, we estimated that there would be a $20 \mathrm{pg} / \mathrm{ml}$ difference in $\mathrm{NfL}$ levels (assuming a standard deviation of $24 \mathrm{pg} / \mathrm{ml}$ ) between troponin and negative positive patients ${ }^{15}$. This required us to recruit 70 patients to provide $80 \%$ power $(p<0.05)$. We sent all available samples for analysis to maximize study power.

As a secondary analysis, a priori we tested whether cytokines previously associated with $\mathrm{NfL}^{7}$ were also associated with troponin positivity. Hence, we tested whether postoperative day 1 changes in IL-10, IL-1beta or IL-8 were higher in troponin positive patients. The p-value was set at $p<0.013$ to account for Bonferroni correction across the three cytokines. As an exploratory analysis, we also undertook Bonferroni corrected analysis across all 10 cytokines.

Multiple logistic regression was conducted to predict postoperative troponin positive status in $\mathrm{R}$ with age, preoperative troponin status, sex, hypertension treatment, coronary artery disease, prior stroke or TIA, vascular surgery (yes/no), intra-operative blood loss and intra-operative hypotension (defined as the area under the curve that is more than $10 \%$ below the preoperative mean arterial pressure). Post-hoc we additionally tested whether adding IL-10 improved this 
model. The same predictors were used to identify predictors of $\mathrm{NfL}$ rises based on splitting the data at the median threshold. This differed slightly from our prior work ${ }^{7}$, due to addition of variables associated with cardiac risk and the use of logistic regression. However, we also checked similar variables predicted rises in $\mathrm{NfL}$ as a continuous variable using linear regression. 


\section{Results}

One patient had a clinically detected postoperative non-ST elevation myocardial infarction with good correspondence of clinical $\left(20.6 \mathrm{ng} \mathrm{ml}^{-1}\right)$ and research $\left(20.4 \mathrm{ng} \mathrm{ml}^{-1}\right)$ troponin values. Preoperatively there were $22 / 114$ patients with detectable troponins (19\%). There was no relationship between preoperative troponin positivity and preoperative NfL values (MannWhitney test $\mathrm{p}=0.875$, data not shown).

Postoperatively there were 34 TPP subjects. TPP were more likely to have a higher NSQIP-D score (Mann-Whitney $\mathrm{p}$-value 0.015 ), and have detectable preoperative troponin levels ( $>40 \mathrm{pg} / \mathrm{ml}$ ) (chi-squared test $p<0.001$ ) from the total available cohort of 100 . There were borderline associations with chronic obstructive pulmonary disease $(p=0.059)$ and vascular surgery $(p=0.055)$. In the cohort of 94 with both troponin and NfL data, TPP were more likely to be preoperative troponin positive (chi-squared test $p<0.001$, Table 1 ). Indeed, patients with detectable preoperative troponins appeared different to be on a different "troponin trajectory" to patients with undetected preoperative levels (Supplementary Figure 1).

\section{Primary Outcome: Associations with putative postoperative neuronal injury}

On postoperative day 1, TPP showed a greater change in NfL from preoperative levels (median TPP 1.63 vs. median TPN $1.34 \mathrm{pg} / \mathrm{ml}$, Mann-Whitney test, $\mathrm{p}=0.024$, Figure 2). Next, we analyzed whether a significant change in troponin (defined as greater than $40 \mathrm{ng} / \mathrm{ml}$ increase from baseline) was associated with a greater rise in NfL. Twenty subjects showed significant troponin rises from preoperative levels. These subjects showed increases in NfL (median TPP $2.75 \mathrm{pg} / \mathrm{ml}$ 
vs. median TPN 1.32 pg/ml, Mann-Whitney test, $p<0.001$, Figure 3). It is worth emphasizing that the clinical significance of these changes is unclear and, despite statistical significance, these rises were smaller than anticipated in our power analysis.

Since we have previously found associations of delirium with $\mathrm{NfL}$, as a secondary outcome, we tested whether TPP were more likely to incur delirium. TPP were not more likely to be delirious (Chi Squared test, $\mathrm{p}=0.133$ ) or have more severe delirium (Mann-Whitney test, $\mathrm{p}=0.078$ ). However, when we analyzed as a significant change in troponin, troponin changes were associated with being delirious ((Chi Squared test, $p=0.002)$ and more severe delirium (MannWhitney test, $p=0.001$ ).

\section{Associations of increased postoperative troponin with changes in preoperative cytokines}

It was recently shown that preoperative systemic inflammation was associated with postoperative myocardial injury. We therefore tested whether preoperative cytokines would be associated with postoperative TPP. However, after Bonferroni correction, none of the 10 cytokines were associated with postoperative TPP $(p<0.974)$. As inflammation has also been associated with chronic cardiovascular disease, we also tested whether any of the preoperative cytokines were associated with preoperative TPP. After FDR correction, IL-2 was significantly associated with preoperative TPP status ( $p=0.049$, Supplementary Figure 2 ). 
We tested whether postoperative rises in cytokines would be associated with TPP status. We plotted the perioperative time courses of $\mathrm{NfL}$ and cytokines over the first four postoperative days

(Figure 3). After normalization to baseline levels, differences in $\mathrm{NfL}$ and cytokines were visually evident on postoperative day 1 . Next, we investigated whether any of the cytokines would be associated with TPP after multiple comparison correction. The change in IL-10 was significantly associated with TPP after FDR correction for 10 tests $(p=0.031)$.

\section{Associations of increased troponin changes from baseline with changes in postoperative cytokines}

Next, we analyzed whether a significant change in troponin (defined as greater than $40 \mathrm{ng} / \mathrm{ml}$ increase from baseline) was associated with rise in inflammatory cytokines. After FDR correction, postoperative day 1 IL-1ra $(p=0.005)$, IL-6 ( $p=0.040)$, IL-8 $(p=0.002)$ and IL-10 ( $p=0.004)$ were associated with rises in troponin (Figure 3).

\section{Associations of increased postoperative troponin with clinical variables}

TPP outcome was tested for association with age, sex, hypertension treatment, CAD, prior stroke or TIA, preoperative troponin status, vascular surgery (yes/no), intra-operative blood loss and intra-operative hypotension using multiple logistic regression. Out of the nine predictor variables, logistic regression showed that TPP was associated with intraoperative hypotension $(p=0.019)$ and preoperative troponin positivity $(p<0.001$, Table 2$)$. 
Given that IL-10 was implicated in both TPP and troponin change analyses above, we conducted linear regression to test whether the clinical predictors may also predict IL-10 concentrations. Intraoperative hypotension $(p=0.010)$, vascular surgery $(p=0.005)$ and blood loss $(p=0.019)$ all predicted IL-10 (Table 2). Notably, preoperative troponin positivity was not associated with rises in IL-10 in this model. These data suggest there is shared variance in intraoperative hypotension and postoperative rises in IL-10.

\section{Associations of increased troponin change with clinical variables}

We conducted logistic regression for the significant change in troponin outcome. For this model we excluded preoperative troponin status (to avoid statistical coupling) but included all other covariates. This model found that more intraoperative hypotension $(p=0.009)$ and blood loss $(p=0.031)$ predicted change in troponin.

\section{Associations of Neurofilament Light with clinical variables}

As the troponin analyses were based on a binary end point (positive yes/no), we binarized the $\mathrm{NfL}$ data based on the median of the data. Logistic regression again showed that preoperative stroke $(p=0.043)$, not incurring vascular surgery $(p=0.020)$ and blood loss $(p=0.002)$, but not intraoperative hypotension were predictive of higher NfL values (Table 2). Linear regression for $\mathrm{NfL}$ as a continuous variable (using the same predictors) suggested associations with the same predictors, preoperative stroke $(p=0.030)$, not having vascular surgery $(p=0.020)$ and blood loss $(p<0.001)$, but not intraoperative hypotension or preoperative troponin positivity (data not shown). Finally, to provide an analogous model for the change in troponin, we investigated the 
predictors of binarized $\mathrm{NfL}$ endpoint with logistic regression but excluding preoperative detectable troponin status. In this model, the same covariates as above were predictive: preoperative stroke $(p=0.042)$, not incurring vascular surgery $(p=0.022)$ and blood loss $(p=0.002)$.

\section{Discussion}

$\mathrm{NfL}$ levels rose on postoperative day 1 to a greater extent in patients with myocardial injury. We showed this using two different definitions of myocardial injury: TPP, detected solely from postoperative detected troponins (Figure 2) or based on the change from preoperative levels

(Figure 3). It is important to stress that, while this was statistically significant in both analyses, the median differences were very small and far smaller than we anticipated. Nonetheless, this suggests that patients who are vulnerable to myocardial injury may also be vulnerable to neuronal injury.

TPP status was associated with increases in IL-10, while the change in troponin was associated with changes in IL-1ra, IL-6, IL-8 and IL-10. These data suggest that postoperative day 1 changes in inflammation may be associated with myocardial injury. Of course, these data do not imply a causal relationship but do suggest there may be some pathological overlap with neuronal injury (which we recently showed was associated with IL-1beta, IL-8 and IL-107). It is of interest that IL10 typically follows myocardial injury, rather than drives $\mathrm{it}^{16}$, and it may act as a cardioprotective rather than injurious agent ${ }^{16}$ (despite clinical associations of IL-10 with adverse cardiac outcomes ${ }^{17}$ ). These data are consistent with our sampling on postoperative day 1 and recent evidence that troponin rises are evident within six hours of surgery ${ }^{5}$. 
In terms of clinical predictors, TPP were more likely to have lower intraoperative blood pressure and be troponin positive at baseline. Intraoperative hypotension and blood loss predicted preoperative to postoperative change in troponin, hence a consistent possible role of intraoperative hypotension was evident in the aetiology of myocardial injury. This represents an important discordance from NfL rises, as intraoperative hypotension was not associated with neuronal injury in our analyses.

Overall, in this cohort, myocardial injury appears to be driven by events on the day of surgery associated with the duration of intraoperative hypotension, as observed previously ${ }^{13}$, as well as predisposition to injury as denoted by detectable preoperative troponin levels (but not preoperative inflammatory cytokines) ${ }^{18}$. Presently it is unclear if inflammatory mechanisms contribute to perioperative myocardial injury following non-cardiac surgery; our data suggest an association and further studies should attempt to manipulate inflammation to reduce the burden of cardiac and neuronal injury. Interestingly, NfL changes were associated with blood loss and not intraoperative hypotension. These data suggest that patients who are vulnerable to myocardial injury may also be vulnerable to neuronal injury, but there are differences in the aetiology (consistent with small differences in the median $\mathrm{NfL}$ values between troponin positive subjects). Importantly, myocardial injury per se does not result in increased release of NfL into the bloodstream (ref: PMID: 32176641). In summary, we suspect that myocardial injury maybe driven by perioperative ischaemia (consistent with a subsequent rise in a cardioprotective IL-10 
response), while neuronal injury requires a breakdown in the blood brain barrier driven by inflammation?.

We strictly adhered to multiple comparison correction, and it is worth noting that with a larger sample size we may have observed significant associations of additional cytokines with TPP or the change in troponin. However, our data were primarily limited by our focus on postoperative sampling. More frequent collection on the day of surgery will be necessary to clarify whether shorter-term inflammatory effects are important in the pathogenesis of early myocardial injury ${ }^{5}$. Also, it is important to note that, myocardial injury may evolve over time, at least in some subjects (Figure 2). It is possible that this may reflect ongoing injury or a secondary hit occurs in the postoperative period in certain vulnerable individuals. Future research should address whether postoperative factors exacerbate the injury incurred on postoperative day 1 . With only one myocardial infarction in this cohort, we are limited in our ability to comment further on the relationship of MINS and perioperative myocardial infarction. It is important to reflect that the single myocardial infarction occurred on postoperative day 2 (not day 1). In this subject, the research troponin value on postoperative day 1 was $1.1 \mathrm{ng} / \mathrm{ml}$ indicating the presence of myocardial injury prior to the clinical non-ST elevation myocardial infarction. This patient appeared to progress from myocardial injury to non-ST elevation myocardial infarction. Future studies should identify factors that affect the transition from MINS to major adverse cardiac events and whether on going inflammation may be a modifiable risk factor for this transition. Another limitation is that, while troponin has a diagnostic value for binarizing a myocardial injury diagnosis, NfL does not yet have a similar, clinically defined, important threshold. Yet, we found 
similar results for linear regression as well as logistic regression using a binarized threshold for $\mathrm{NfL}$ changes. This reassures us that the differences observed were not just due to differences in the analytic approach.

\section{Conclusions}

In summary our data suggest that patients who are vulnerable to MINS may also be vulnerable to neuronal injury. However, it is important to note that the differences in NfL levels were subtle. Further, the differing associations with intraoperative hypotension suggest there may be important differences in the underlying aetiologies of cardiac and neuronal injury.

\section{Details of authors' contributions}

RDS designed the research with input from RL and RAP. RDS, LC, CC, MW, MP, AB, TB all collected data. Data analysis was conducted by RDS, CC, HZ and KB, and TB. All authors contributed to interpretation and writing the article.

\section{Acknowledgements}

$\mathrm{HZ}$ is a Wallenberg Scholar supported by grants from the Swedish Research Council (\#201802532), the European Research Council (\#681712), Swedish State Support for Clinical Research (\#ALFGBG-720931), the Alzheimer Drug Discovery Foundation (ADDF), USA (\#201809-2016862), and the UK Dementia Research Institute at UCL.

\section{Declaration of interests}

$\mathrm{HZ}$ has served at scientific advisory boards for Denali, Roche Diagnostics, Wave, Samumed, Siemens Healthineers, Pinteon Therapeutics and $\operatorname{CogRx}$, has given lectures in symposia sponsored by Fujirebio, Alzecure and Biogen, and is a co-founder of Brain Biomarker Solutions in Gothenburg AB (BBS), which is a part of the GU Ventures Incubator Program (outside submitted work). The other authors report no conflict of interest.

\section{Funding}

RDS, RL and RAP are supported by R01 AG063849-01. RDS is supported by 1R01NS117901-01. 
Figure 1. STROBE Diagram.

Figure 2. Time course of perioperative changes in biomarkers for troponin positive (red) or negative (blue) patients based solely on postoperative troponin values. Data are normalized to preoperative values and for the purposes of display only are shown as mean plus standard error of the mean across all panels.

Figure 3. Time course of perioperative changes in biomarkers for patients with significant troponin changes from preoperative levels (red) or not (blue). Data are normalized to preoperative values and for the purposes of display only are shown as mean plus standard error of the mean across all panels.

Table 1. Demographics.

Table 2. Regression Models for troponin positive patients (logistic), change in IL-10 (linear) and neurofilament light $(\mathrm{NfL})$ levels (logistic).

Supplementary Figure 1. Non-normalized Time course of perioperative changes in biomarkers for troponin positive (red) or negative (blue) patients based solely on postoperative troponin values. Data are not normalized to preoperative values.

Supplementary Figure 2. Preoperative IL-2 is associated with detectable preoperative troponins (>40 pg/ml).

\section{References}


1. Puelacher C, Lurati Buse G, Seeberger D, et al. Perioperative Myocardial Injury After Noncardiac Surgery: Incidence, Mortality, and Characterization. Circulation 2018;137:1221-32.

2. Croal BL, Hillis GS, Gibson PH, et al. Relationship between postoperative cardiac troponin I levels and outcome of cardiac surgery. Circulation 2006;114:1468-75.

3. Devereaux PJ, Chan MT, Alonso-Coello P, et al. Association between postoperative troponin levels and 30-day mortality among patients undergoing noncardiac surgery. JAMA : the journal of the American Medical Association 2012;307:2295-304.

4. Noordzij PG, van Geffen O, Dijkstra IM, et al. High-sensitive cardiac troponin T measurements in prediction of non-cardiac complications after major abdominal surgery. Br J Anaesth 2015;114:909-18.

5. Ackland GL, Abbott TEF, Jones TF, et al. Early elevation in plasma high-sensitivity troponin $T$ and morbidity after elective noncardiac surgery: prospective multicentre observational cohort study. Br J Anaesth 2020;124:535-43.

6. May SM, Abbott TEF, Del Arroyo AG, et al. MicroRNA signatures of perioperative myocardial injury after elective noncardiac surgery: prospective observational mechanistic cohort study. Br J Anaesth 2020.

7. Casey $\mathrm{CP}$, Lindroth $\mathrm{H}$, Mohanty $\mathrm{R}$, et al. Postoperative delirium is associated with increased plasma neurofilament light. Brain 2019;143:47-54.

8. Neuro VI. Perioperative covert stroke in patients undergoing non-cardiac surgery (NeuroVISION): a prospective cohort study. Lancet 2019;394:1022-9.

9. Ackland GL, Abbott TEF, Cain D, et al. Preoperative systemic inflammation and perioperative myocardial injury: prospective observational multicentre cohort study of patients undergoing non-cardiac surgery. Br J Anaesth 2019;122:180-7.

10. Hunter JD, Doddi M. Sepsis and the heart. Br J Anaesth 2010;104:3-11.

11. Landis RC, Brown JR, Fitzgerald D, et al. Attenuating the Systemic Inflammatory Response to Adult Cardiopulmonary Bypass: A Critical Review of the Evidence Base. J Extra Corpor Technol 2014;46:197-211.

12. van Waes JA, van Klei WA, Wijeysundera DN, van Wolfswinkel L, Lindsay TF, Beattie WS. Association between Intraoperative Hypotension and Myocardial Injury after Vascular Surgery. Anesthesiology 2016;124:35-44.

13. Abbott TEF, Pearse RM, Archbold RA, et al. A Prospective International Multicentre Cohort Study of Intraoperative Heart Rate and Systolic Blood Pressure and Myocardial Injury After Noncardiac Surgery: Results of the VISION Study. Anesth Analg 2018;126:1936-45.

14. Gisslen M, Price RW, Andreasson U, et al. Plasma Concentration of the Neurofilament Light Protein (NFL) is a Biomarker of CNS Injury in HIV Infection: A Cross-Sectional Study. EBioMedicine 2016;3:135-40.

15. Evered L, Silbert B, Scott DA, Zetterberg H, Blennow K. Association of Changes in Plasma Neurofilament Light and Tau Levels With Anesthesia and Surgery: Results From the CAPACITY and ARCADIAN Studies. JAMA Neurol 2018;75:542-7.

16. Jung $M, M a Y$, lyer RP, et al. IL-10 improves cardiac remodeling after myocardial infarction by stimulating M2 macrophage polarization and fibroblast activation. Basic Res Cardiol 2017;112:33. 
17. Welsh P, Murray HM, Ford I, et al. Circulating interleukin-10 and risk of cardiovascular events: a prospective study in the elderly at risk. Arterioscler Thromb Vasc Biol 2011;31:233844.

18. Zhao BC, Liu WF, Deng QW, et al. Meta-analysis of preoperative high-sensitivity cardiac troponin measurement in non-cardiac surgical patients at risk of cardiovascular complications. The British journal of surgery 2020;107:e81-e90. 